\title{
Granular compaction and the topology of pore deformation
}

\author{
Mohammad Saadatfar ${ }^{1, \star}$, Hiroshi Takeuchi ${ }^{2}$, Maryam Hanifpour ${ }^{3}$, Vanessa Robins ${ }^{1}$, Nicolas Francois ${ }^{1}$, and Yasuaki \\ Hiraoka ${ }^{2}$ \\ ${ }^{1}$ Department of Applied Mathematics, Research School of Physics and Engineering, The Australian National University, Canberra \\ ACT 2601, Australia \\ ${ }^{2}$ Graduate School of Science, Tohoku University. 6-3, Aramaki Aza-Aoba, Aoba-ku, Sendai, 980-8578, Japan \\ ${ }^{3}$ Department of Applied Physics, Aalto University, Helsinki
}

\begin{abstract}
The mechanism of crystallisation in highly dissipative materials such as foams or granular materials is still widely unknown. In macroscopic granular materials high levels of energy need to be injected to overcome the natural propensity of these dissipative materials to form amorphous structures [1,2]. The transition from disordered to ordered packings in such systems triggers a wide range of geometrical, topological and mechanical changes at multi length scales [3]. Formation of cavities and patterns by aggregates of grains and their evolution during this transition requires a complete topological description of the system. Here, crystallisation of three-dimensional packings of frictional spheres is studied at the grain scale with $\mathrm{x}$-ray tomography. Using a novel and powerful topological tool, Persistent Homology, we describe the complete formation process of perfect tetrahedral and octahedral patterns: the two building blocks of FCC and HCP crystalline arrangements. Additionally we present possible and allowable deformations of these components that accurately reproduce the main topological features of the system. These results give new insights into the crystallisation of these highly dissipative materials.
\end{abstract}

\section{Introduction}

A granular assembly is comprised of numerous particles therefore amenable to statistical mechanics approach. However, such an approach has proven to be extremely challenging due to the fact that the physics of granular systems are inherently different at micro and macro scales. On one end of the granular matter spectrum is the thermal microscopic systems, including foams, colloids, and molecular glass formers, where glassy behaviour is observed. Crystallisation in these systems are described by equilibrium statistical mechanics, where their phase space can be fully described via entropic interactions.

At the other end of the spectrum are the macroscopic (mesoscopic) granular systems, which are athermal and frictional and therefor far from thermodynamic equilibrium. In these out-of-equilibrium systems the granular particles are so heavy that their thermal motion is negligible. Due to the presence of friction at this length scale, the inter-particle interaction is dissipative and the kinetic energy of the particles is irrecoverably lost to microscopic degrees of freedom. However, if a macroscopic hardsphere packing is mechanically stable (e.g at rest under gravity), an analogy with equilibrium statistical mechanics can be drawn, where one assumes that all microstates (mechanically stable states) occur with the same probability . In Edwards' proposal to describe a macroscopic granular

\footnotetext{
^e-mail:mos110@physics.anu.edu.au
}

assembly using thermodynamics that is analogous to thermal systems, the volume of granular packing replaces the energy. In this approach, the configurational space (grain positions and their orientational arrangements in the packing) and the local free volume available to each grain play a crucial role [4-8].

In this paper we demonstrate that new ideas associated with computational topology provide an efficient, robust and faithful approach to implementing tractable models to decipher the complexity of the spatial structures of the configurational space of granular systems. More specifically, we present a novel topological characterisation tool, Persistent Homology (PH), to study dense granular systems. We show that $\mathrm{PH}$ is able to explore and characterise the configurational phase space of disordered and partially ordered macroscopic granular systems by identifying key features specific to granular systems. We first detail the experimental systems followed by the mathematical description of the persistent homology, and in particular its application for granular systems.

\section{Experimental procedure}

We analyse two sets of experimental granular packings each containing over 150,000 monosized acrylic beads (diameter $\mathrm{d}=1 \mathrm{~mm}$, polydispersity $=0.025 \mathrm{~mm}$ ): partially ordered cylindrical (see Fig. 1.a) and spherical (see Fig. 1.b) packing produced using a vibrational protocol with similar packing densities of $\phi \approx 0.68$ Details 

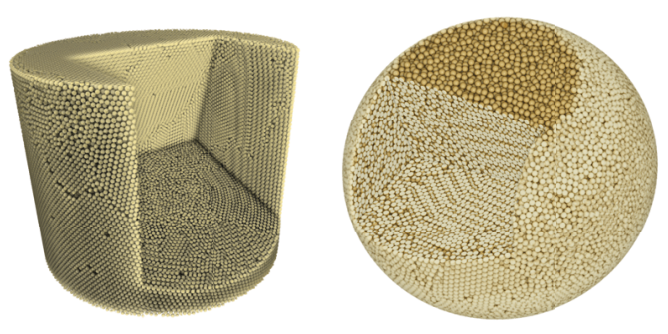

Figure 1. 3D visualisation of the internal structure of partially crystallised packing with a global packing density of $\phi=0.685$ in (a) a cylindrical container, (b) a spherical container.

of the experimental procedure can be found elsewhere $[2,9]$. Our experiments harness X-ray Computed Tomography (XCT) and three-dimensional (3D) image analysis to accurately determine grain centres with the precision of $\left(<10^{-3} \mu \mathrm{m}\right)$ and grain's diameter with precisions greater than $\approx 10^{-2} \mu m[3]$. Figure $1(\mathrm{a} \& \mathrm{~b})$ show $3 \mathrm{D}$ renderings of such partially crystallised structures.

\subsection{Persistant Homology}

Homology is an algebraic tool for studying topological structures. Persistent Homology $(\mathrm{PH})$ is a technique that facilitates the quantification of topological structures in data [10]. Persistent homology extends traditional homology by tracking how the homology groups change as an object grows. Each homology class has two values, which are calculated by varying a filtration parameter associated with it: a birth value and a death value. It is common practice to represent this information in a Persistence Diagram (PD) for each dimension of homology. $\mathrm{PD}_{k}$ contains all pairs $(b, d), b \leq d$, associated with a persistent homology class in dimension $k$.

For a perfectly mono-disperse bead pack with no "rattlers", all points in $\mathrm{PD}_{0}$ have birth $=0$ and death $=r$. Each point in $\mathrm{PD}_{2}$ represents a kind of pore or cavity in the interstices between the beads. Perfectly crystalline sphere packings consist of a periodic arrangement of tetrahedral and octahedral cavities. This close packing configuration has a maximal packing density of $\phi=0.74$. Below the RCP limit $(\phi=0.64)$, a great number of cavities are confined within a curved strip, one end of which is pointing to the the location of the tetrahedral coordinate in PD2. We note the presence of a less populated region located at higher death values $(d>\sqrt{2} \times D)$ with a peak around $(b, d)=(0.04,0.07)$ as seen in Figure 2.a. This region is a characteristic of packings containing amorphous structures with cavities that are irregular and larger than octahedral pores. Each point, $(b, d)$, in this region of PD2 represents a connected chain of many pores, with the death value being the radius of the largest pore in the chain, and the birth value being the maximal throat (3-bead contact radius) that separates the extended pore from the others.

\section{Cavity formation/deformation scenarios}

\section{A: Tetrahedral deformations}

We consider two types of tetrahedral deformations, which are permitted in a close bead packing. The first tetrahedral deformation, D1, consists of letting one bead of a tetrahedron roll across the saddle formed by two neighbours, while the other beads stay fixed. A top view of this deformation (D1) is demonstrated in Figure2.b and the $P D_{2}$ associated curve produced by this deformation can be seen in Figure2.a. The second tetrahedral deformation, D2, is a symmetric process that transforms a regular tetrahedron into a flat square configuration. A top view of this deformation in shown in Figure2.b. The PD2 curves of this deformation can be seen in Figure2.a.

D1\&D2 curves run along the lower and upper boundaries of a densely populated region in $P D_{2}$, where they are joined at the location of tetrahedral cavity (Figure2.a).

The curves D1 and D2 clearly delineate the highly populated strip which we identify as a region made of weakly distorted tetrahedral cavities. The extent of this domain confirms the variety and ubiquity of weakly distorted tetrahedra in partially crystallised packings It has recently been shown that the formation of polytetrahedral aggregates composed of weakly distorted tetrahedra is a geometrical principle of densification for amorphous frictional packings and a resilient feature in partially crystallised structures up to $\phi=0.73[2,11]$.

\section{B: Octahedral deformation}

Here we consider two relevant octahedral deformations each closely matching one of the densely populated paths connecting the octahedral hotspot to the diagonal line in $P D_{2}$. These dense regions are especially pronounced in the $P D_{2}$ of the partially crystallised packing $(\phi \approx 0.68 \%)$ as seen in Figure 2.c.

The first one is simply lengthening two opposite edges of the octahedron (symmetrically), while keeping all other edges equal to $2 r$. This deformation scenario, D3, transforms two edge-adjacent tetrahedra into a regular octahedron as shown in the top cartoon in Figure 2.b. The second octahedral deformation, D4, is similar to the first tetrahedral deformation (D1), where only one of the beads roll along the saddle. This scenario is essentially the repeat of D1 deformation for an octahedral grain configuration as demonstrated in Figure 2.b. The birth-death curves of the above four deformation scenarios are superimposed on the $P D_{2}$ of our partially crystallised packing with the density of $\phi \approx 0.68$ ( Figure 2.a). Moreover, D1-D4 curves and the evolution of $(b, d)$ coordinates of these regular cavity deformations can be derived analytically using a combination of dihedral angles and grain radius [12].

\section{Formation scenarios of regular cavities and mechanical features of packing crystallisation.}

The above four formation mechanisms of regular cavities allow us to describe most of the evolution of $\mathrm{PD}_{2}$ over a 

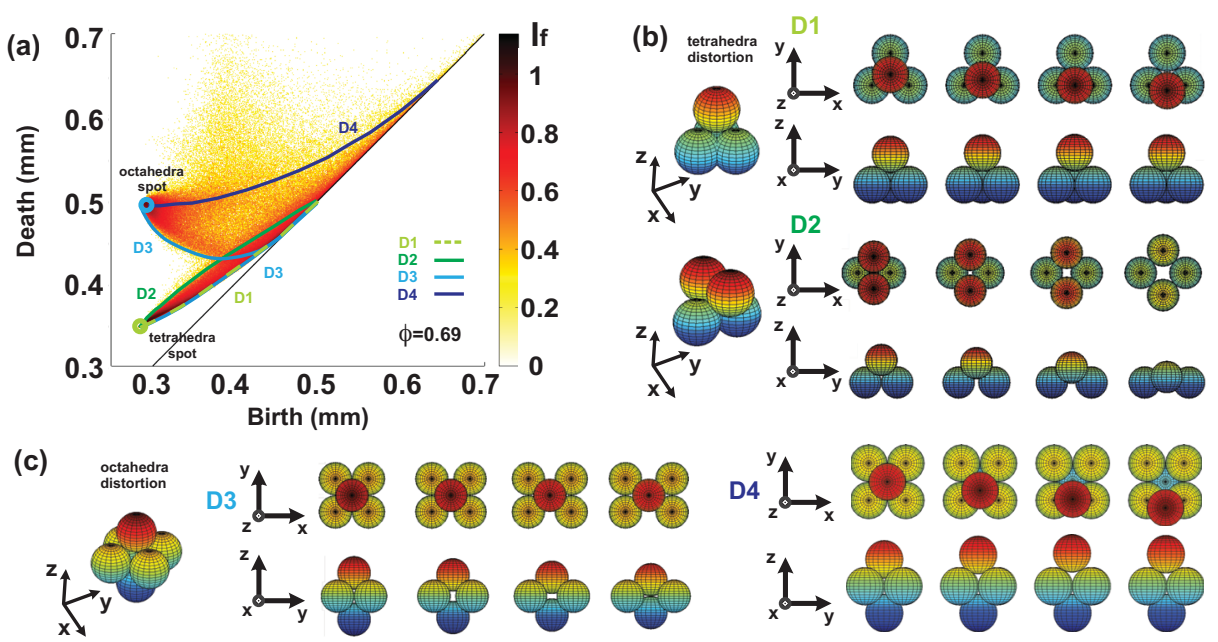

Figure 2. (a) $\mathrm{PD}_{2}$ of a partially crystallised sphere packing with density $\phi=0.685$. The superimposed curves correspond to analytically computed birth-death curves of the deformation scenarios shown in (b) and (c). (b) Top and side views of D1 and D2 deformations scenarios of a tetrahedral cavity. (c) Top and side views of D3 and D4 deformation scenarios of an octahedral cavity. The color code indicates the relative height of sections of the grain with respect to horizontal median plane.

broad density range $0.64<\phi<0.73$. This predictive power is quite remarkable given that these mechanisms are based on idealised deformation of regular cavities made of identical grains.

To gain more insight into this matter, let us now consider the four mechanisms depicted in Figs. 2(b,c) from a mechanical viewpoint. More precisely, we are interested in the mechanical contacts between those grains: i.e. the internal contacts within a specific motif made of $\mathrm{N}$ grains. In the following, we assume that the number of mechanical contacts surrounding the motif (i.e. the external contacts) remain unchanged. Table 1 shows the total number of internal mechanical contacts $\Delta C_{i n t}$ created during the formation of regular motifs according to the four scenarios. For each mechanism the number of contacts created per grain, $\Delta C_{\text {int }} \cdot 2 / N$, is between $1 / 2$ and 1 . This is an important feature of the formation mechanisms chosen: a small number of contacts is modified. To support this statement, we have tested other deformation mechanisms that allow more internal contacts to be modified and none of them describe accurately the evolution of $\mathrm{PD}_{2}$.

The mechanical stability of packings depends on both mechanical and geometrical characteristics of the neighbourhood of each grain [1, 13-15]. This feature of pack-

Table 1. Number of internal mechanical contacts $\Delta C_{\text {int }}$ created during the formation of tetrahedral and octahedral cavities according to the scenarios D1, D2, D3, D4. N is the number of beads involved in the pattern. $C_{\text {intfinal }}$ is the total number of contacts in the final regular pattern. To define the number of contact created per grain, we divide $\Delta C_{\text {int }}$ by $N / 2$ to account for the fact that a contact links two grains.

\begin{tabular}{lcccc} 
Scenario & $\mathrm{N}$ & $C_{\text {intfinal }}$ & $\Delta C_{\text {int }}$ & $\Delta C_{\text {int }} \cdot 2 / N$ \\
\hline D1 & 4 & 6 & 1 & $1 / 2$ \\
\hline D2 & 4 & 6 & 2 & 1 \\
\hline D3 & 6 & 12 & 2 & $2 / 3$ \\
\hline D4 & 6 & 12 & 2 & $2 / 3$ \\
\hline
\end{tabular}

ing stability has a huge influence on the crystallisation process [3]. One of the most remarkable consequences is the existence of a plateau for the mechanical contact number $<Z_{m}>$ (i.e. the average number of contact per grain) over the density range $0.64<\phi<0.68$. This feature has been observed in numerical simulations of frictionless spheres and experimentally in the case of frictional packings [3, 16, 17]. Quantitatively, $\left\langle Z_{m}>\right.$ is constant at $<Z_{m}>\approx 5.6$ over the density range $0.64<\phi<0.68$, beyond $\left\langle Z_{m}>\right.$ increases again and reaches $\left\langle Z_{m}>\approx 6.8\right.$ at $\phi=0.73$, a value well below $<Z_{m}>=12$ as expected for perfect FCC or HCP crystals [3]. Therefore almost-crystalline packings made of weakly polydisperse beads still possesses a highly random mechanical backbone with properties close to that of amorphous packings $[3,18-20]$. Quantitatively, there is a relatively good match between the values of $\Delta C_{i n t} \cdot 2 / N$ and the fact that actual measurements of $\left\langle Z_{m}>\right.$ show an increase of 1.2 over $0.64<\phi<0.73[3,17]$.

There is no doubt that the deformation scenarios presented here are idealised (no grain polydispersity, no influence of the second shell of grains), however, they highlight essential features of crystallisation in granular packings:i) a small number of mechanical contacts per grain is created during the crystallisation. ii) since the mechanical structure of highly crystalline packing made of weakly polydisperse beads shares some similarities with that of amorphous packings, grain-scale structural evolution in the first shell of neighbours (rather than global periodic conditions $[16,21]$ ) is expected to remain of prime importance to characterise packing stability $[3,13]$. iii) the distorted cavities at the boundaries of the existence domain in $\mathrm{PD}_{2}$ have to be dense configuration of grains, a natural consequence of crystallisation as a densification process based on the creation of tetrahedral and octahedral cavities. 


\section{Conclusions}

Although persistent homology is a geometrical characterisation, we emphasise that the experimental nature of our packings ensures that the diagrams presented here actually describe the space of cavities that not only exist but are also mechanically stable. To illustrate this, we have identified four grain-scale deformation mechanisms that recover prominent features of the diagram evolution and highlight basic grain-scale rearrangements, pattern selection, and mechanical constraints underpinning packing crystallisation. We note that connections between topological features of packings and their mechanical stability have recently been uncovered [22]. The results reported here represent a basis on which to interpret at the grainscale why granular crystallisation, an out-equilibrium phenomenon in a complex system can be mapped onto an established framework of statistical mechanics [16]. Our findings have also practical applications in domains such as pore description in soil- and geo-sciences, which are crucial for understanding natural systems mechanical stability, vibration induced compaction and flow permeability [23-25].

\section{References}

[1] Wyart, M. Marginal stability constrains force and pair distributions at random close packing. Phys. Rev. Lett. 109, 125502 (2012).

[2] Francois, N., Saadatfar, M., Cruikshank, R. \& Sheppard, A. Geometrical frustration in amorphous and partially crystallized packings of spheres. Phys. Rev. Lett. 111, 148001 (2013).

[3] Hanifpour, M., Francois, N., Vaez Allaei, S. M., Senden, T. \& Saadatfar, M. Mechanical characterization of partially crystallized sphere packings. Phys. Rev. Lett. 113, 148001 (2014).

[4] Song, C., Wang, P. \& Makse, H. A. A phase diagram for jammed matter. Nature 453, 629-632 (2008).

[5] Blumenfeld, R., Jordan, J. F. \& Edwards, S. F. Interdependence of the volume and stress ensembles and equipartition in statistical mechanics of granular systems. Phys. Rev. Lett. 109, 238001 (2012).

[6] McNamara, S., Richard, P., Kiesgen de Richter, S., Le Caër G. \& Delannay, R. Measurement of granular entropy. Phys. Rev. E 80, 031301 (2009).

[7] Puckett, J. G. \& Daniels, K. E. Equilibrating temperaturelike variables in jammed granular subsystems. Phys. Rev. Lett. 110, 058001 (2013).

[8] Ashwin, S. S., Blawzdziewicz, J., O’Hern, C. S. \& Shattuck, M. D. Calculations of the structure of basin volumes for mechanically stable packings. Phys. Rev. E 85, 061307 (2012).

[9] Aste, T., Saadatfar, M. \& Senden, T. J. Geometrical structure of disordered sphere packings. Phys. Rev. E 71, 061302 (2005).
[10] Robins, V., Wood, P. J. \& Sheppard, A. P. Theory and algorithms for constructing discrete Morse complexes from grayscale digital images. Pattern Analysis and Machine Intelligence, IEEE Transactions, 33, 1646-1658 (2011).

[11] Anikeenko, A. V., Medvedev, N. N. \& Aste, T. Structural and entropic insights into the nature of the random-close-packing limit. Phys. Rev. E 77, 031101 (2008).

[12] Saadatfar, M., Takeuchi, H., Robins, V., Francois, N., \& Yasuaki, H. Topological configuration landscape and pore deformation mechanisms during the crystallisation of sphere packing. Nature Communications, (2016.)

[13] van Hecke, M. Jamming of soft particles: geometry, mechanics, scaling and isostaticity. J. of Physics: Condensed Matter 22, 033101 (2010).

[14] Charbonneau, P., Corwin, E. I., Parisi, G. \& Zamponi, F. Universal microstructure and mechanical stability of jammed packings. Phys. Rev. Lett. 109, 205501 (2012).

[15] O’Hern, C. S., Langer, S. A., Liu, A. J. \& Nagel, S. R. Force distributions near jamming and glass transitions. Phys. Rev. Lett. 86, 111 (2001).

[16] Jin, Y. \& Makse, H. A. A first-order phase transition defines the random close packing of hard spheres. Physica A 389, 5362 (2010).

[17] Hanifpour, M., Francois, N., Allaei, S. V., Senden, T. \& Saadatfar, M. Structural and mechanical features of the order-disorder transition in experimental hardsphere packings. Phys. Rev. E 91, 062202 (2015).

[18] Mari, R., Krzakala, F. \& Kurchan, J. Jamming versus glass transitions. Phys. Rev. Lett. 103, 025701 (2009).

[19] Goodrich, C. P., Liu, A. J. \& Nagel, S. R. Solids between the mechanical extremes of order and disorder. Nature Physics 10, 578 (2014).

[20] Tong, H., Tan, P. \& Xu, N. From Crystals to Disordered Crystals: A Hidden Order-Disorder Transition. Scientific Reports 5, doi:10.1038/srep15378 (2015).

[21] Moukarzel, C. F. Isostatic phase transition and instability in stiff granular materials. Phys. Rev. Lett. 81, 1634 (1998).

[22] Carlsson, G., Gorham, J., Kahle, M. \& Mason, J. Computational topology for configuration spaces of hard disks. Phys. Rev. E 85, 011303 (2012).

[23] Saadatfar M. et al., 3D mapping of deformation in an unconsolidated sand: A micro mechanical study. SEG Tech. Prog. Expan. Abst. 2012, 1 (2012).

[24] Robins, V., Saadatfar, M., Delgado-Friedrichs, O. \& Sheppard, A. P. Percolating length scales from topological persistence analysis of micro-CT images of porous materials. Water Resources Research, DOI: 10.1002/2015WR017937 (2016).

[25] Sufian, A., Russell, A. R., Whittle, A. J. \& Saadatfar, M., Pore shapes, volume distribution and orientations in monodisperse granular assemblies. Granular Matter, 17(6), 727-742 (2015). 